PROCEEDINGS OF THE

AMERICAN MATHEMATICAL SOCIETY

Volume 139, Number 2, February 2011, Pages 583-595

S 0002-9939(2010)10659-7

Article electronically published on August 26, 2010

\title{
THE LANDAU THEOREM AND BLOCH THEOREM FOR PLANAR HARMONIC AND PLURIHARMONIC MAPPINGS
}

\author{
HUAIHUI CHEN AND PAUL M. GAUTHIER
}

(Communicated by Mario Bonk)

\begin{abstract}
For a normalized quasiregular pluriharmonic mapping $f$ of the unit ball $B^{n}$ of $\mathbb{C}^{n}$ into $\mathbb{C}^{n}$, we estimate the supremum of numbers $R$ such that some subdomain $\Omega$ of the ball is mapped by $f$ diffeomorphically onto some ball of radius $R$. Our estimates significantly improve earlier estimates, even in the case of harmonic functions in the disc.
\end{abstract}

\section{INTRODUCTION}

The classical Bloch theorem asserts the existence of a positive absolute constant $b$ such that for any holomorphic mapping $f$ of the unit disk $D$, with the normalization $f^{\prime}(0)=1$, the image $f(D)$, as a covering surface, contains a univalent disk of radius $b$. Bloch's constant is defined as the supremum of such constants $b$.

In 1990, Mario Bonk [3] improved Ahlfors' lower estimate of Bloch's constant which had prevailed for more that fifty years. It has been said that finding the precise value of the Bloch constant replaced the Bieberbach conjecture as the most important open problem in function theory, after the latter was confirmed by Louis de Branges in 1985. At the present time the best lower bound of Bloch's constant is $\frac{\sqrt{3}}{4}+0.0002$, which is due to the authors of the present paper [5].

For planar harmonic mappings, in order to obtain a Bloch theorem, some extra restriction, other than the normalization at the origin, must be added. For $K$-quasiregular harmonic mappings (even in higher dimensions), Bochner 2] had already proved the existence of a Bloch constant, but gave no estimate. In [7, a concrete lower bound of the Bloch constant for planar $K$-quasiregular harmonic mappings was given by us together with Hengartner; moreover, it is proved there that the Bloch theorem is valid for open harmonic mappings. Related to the Bloch theorem, the Landau theorem for planar harmonic mappings was established in the same paper. In the case of several variables, we proved a Bloch theorem for quasiregular holomorphic mappings in another paper [6].

Received by the editors March 11, 2010.

2010 Mathematics Subject Classification. Primary 30C99; Secondary 30C62.

Key words and phrases. Bloch constant, harmonic mappings.

This research was supported in part by NSFC (China, Grant No. 10671093) and NSERC (Canada).

(C)2010 American Mathematical Society Reverts to public domain 28 years from publication 
In the present paper, we continue this investigation. First, we give significant improvements of the lower bounds of the Bloch constants for $K$-quasiregular harmonic mappings and for open harmonic mappings. Then, we consider pluriharmonic mappings in higher dimensions and establish the Schwarz-Pick lemma and Landau theorem for pluriharmonic mappings as well as the Bloch theorem for pluriharmonic $K$-mappings.

\section{PlanAR HARMONIC MAPPINGS}

For a continuously differentiable complex-valued function $f(z)=u(z)+i v(z)$, $z=x+i y$, we use the common notation for its formal derivatives:

$$
f_{z}=\frac{1}{2}\left(f_{x}-i f_{y}\right), \quad f_{\bar{z}}=\frac{1}{2}\left(f_{x}+i f_{y}\right)
$$

Then $f$ is a harmonic mapping if and only if $f$ is twice continuously differentiable and $\Delta f=4 f_{z \bar{z}}=0$.

Let $f$ be a harmonic mapping of a domain $G$. $f$ is said to be univalent or locally univalent if $f$ is one-to-one or locally one-to-one on $G$. It is known 12 that a harmonic mapping is locally univalent if and only if the determinant $\operatorname{det} J_{f}$ of its Jacobian matrix $J_{f}$ does not vanish anywhere. Such a result does not hold in higher dimensions [17]. Locally, $f$ can be written as $f=\bar{g}+h$, where $g$ and $h$ are holomorphic. Since $f_{z}=h^{\prime}$ and $f_{\bar{z}}=\overline{g^{\prime}}$, we see that $f_{z}$ and $\overline{f_{\bar{z}}}$ are holomorphic and that

$$
\operatorname{det} J_{f}=u_{x} v_{y}-u_{y} v_{x}=\left|f_{z}\right|^{2}-\left|f_{\bar{z}}\right|^{2}=\left|h^{\prime}\right|^{2}-\left|g^{\prime}\right|^{2} \text {. }
$$

A mapping of a domain $G \subset \mathbb{C}$ is said to be open if it maps any open subset of $G$ to an open set in $\mathbb{C}$. A mapping is said to be light if no continuum (connected closed set containing more than one point) is mapped to a single point. A continuous mapping of a domain in the plane is called an interior transformation in the sense of Stoïlow if $f$ is both open and light. The famous Stoïlow theorem [16] says that an interior transformation $f$ is topologically holomorphic, that is, $f$ can be written in the form $f=F \circ \phi$, where $F$ is a non-constant holomorphic mapping and $\phi$ is a homeomorphism. An interior transformation $f$ is said to be sense-preserving if the homeomorphism $\phi$ is sense-preserving. It is proved in [7] that for harmonic mappings, the openness implies the lightness; i.e., an open harmonic mapping is an interior transformation.

For a continuously differentiable mapping $f$, denote

$$
\begin{aligned}
& \Lambda_{f}=\max _{0 \leq \theta \leq 2 \pi}\left|e^{i \theta} f_{z}+e^{-i \theta} f_{\bar{z}}\right|=\left|f_{z}\right|+\left|f_{\bar{z}}\right|, \\
& \lambda_{f}=\min _{0 \leq \theta \leq 2 \pi}\left|e^{i \theta} f_{z}+e^{-i \theta} f_{\bar{z}}\right|=|| f_{z}|-| f_{\bar{z}}|| .
\end{aligned}
$$

$\Lambda_{f}$ and $\lambda_{f}$ are the maximum dilation and minimum dilation of the mapping $f$ respectively. Note that $\left|\operatorname{det} J_{f}\right|=\Lambda_{f} \lambda_{f} . f$ is said to be $K$-quasiregular $(K \geq 1)$ on a domain $G$ if $\Lambda_{f} \leq K \lambda_{f}$ holds everywhere on $G$.

Theorem 1. A non-constant $K$-quasiregular harmonic mapping is an interior transformation in the sense of Stoïlow. 
Proof. Let $f$ be a $K$-quasiregular harmonic mapping of a domain $G$, which is not a constant. For $z_{0} \in G, f$ has an expansion around $z_{0}$ :

$$
f(z)=f\left(z_{0}\right)+\sum_{n=k}^{\infty} a_{n}\left(z-z_{0}\right)^{n}+b_{n}\left(\bar{z}-\bar{z}_{0}\right)^{n},
$$

where $k \geq 1$ and one of $a_{k}$ and $b_{k}$ is not zero. $\left|a_{k}\right|$ and $\left|b_{k}\right|$ cannot be equal. Otherwise, we may assume that neither $f_{z}$ nor $f_{\bar{z}}$ vanishes identically, so there is a sequence $z_{j} \rightarrow z$ with $\left|f_{z}\left(z_{j}\right) / f_{\bar{z}}\left(z_{j}\right)\right| \rightarrow 1$ and $\lambda_{f}\left(z_{j}\right) / \Lambda_{f}\left(z_{j}\right) \rightarrow 0$ as $z_{j} \rightarrow z_{0}$, which contradicts the quasiregularity.

Without loss of generality, assume that $\left|a_{k}\right|>\left|b_{k}\right|$. Write $f(z)-f\left(z_{0}\right)=h(z)+$ $g(z)$ with

$$
h(z)=a_{k}\left(z-z_{0}\right)^{k}, \quad g(z)=\sum_{n=k+1}^{\infty} a_{n}\left(z-z_{0}\right)^{n}+\sum_{n=k}^{\infty} b_{n}\left(\bar{z}-\bar{z}_{0}\right)^{n} .
$$

For each $r$ sufficiently small, there is a small $\delta>0$ so that $|h(z)|>|g(z)|+\delta$ for $z \in \gamma=\left\{z:\left|z-z_{0}\right|=r\right\}$. Then, for $w^{\prime}$ with $\left|w^{\prime}-f\left(z_{0}\right)\right|<\delta$, we have $\left|g(z)+f\left(z_{0}\right)-w^{\prime}\right|<|g(z)|+\delta<|h(z)|$ for $z \in \gamma$ and, consequently,

$$
\Delta_{\gamma} \arg \left(f(z)-w^{\prime}\right)=\Delta_{\gamma} \arg \left(h(z)+g(z)+f\left(z_{0}\right)-w^{\prime}\right)=\Delta_{\gamma} \arg h(z)=2 k \pi .
$$

Thus, there is at least one zero of $f(z)-w^{\prime}$ in the disk bounded by $\gamma$. This shows that $f(D)$ contains the disk of center $f\left(z_{0}\right)$ and radius $\delta$. Thus, $f$ is open and, consequently, $f$ is an interior transformation in the sense of Stoillow. The theorem is proved.

\section{The harmonic Bloch constants}

For a non-constant harmonic mapping $f$ of the unit disk $D$, a univalent disk contained in $f(D)$ is defined as a disk which is the homeomorphic image of some region in $D$. The supremum $b_{f}$ of the radii of all univalent disks is called the inradius of $f(D)$ and denoted by $b_{f}$. The class of all $K$-quasiregular harmonic mappings of $D$ is denoted by $\operatorname{Har}_{K}(D)$, and that of all open harmonic mappings of $D$ is denoted by $\operatorname{Har}_{O}(D)$. The Bloch constants for $K$-quasiregular harmonic mappings and open harmonic mappings are defined by

$$
\begin{gathered}
\beta_{\text {har }}^{K}=\inf \left\{b_{f}: f \in \operatorname{Har}_{K}(D), \lambda_{f}(0)=1\right\}, \\
B_{h a r}^{K}=\inf \left\{b_{f}: f \in \operatorname{Har}_{K}(D), f_{z}(0)=1,\left|f_{\bar{z}}(0)\right| \leq(K-1) /(K+1)\right\},
\end{gathered}
$$

and

$$
B_{\text {har }}^{O}=\inf \left\{b_{f}: f \in \operatorname{Har}_{O}(D), f_{z}(0)=1, f_{\bar{z}}(0)=0\right\} .
$$

It is obvious that $\beta_{\text {har }}^{K} \geq B_{\text {har }}^{K}$. With this notation the results in [7] are formulated by

$$
\beta_{h a r}^{K} \geq \frac{\pi}{8 \sqrt{2}(1+2 K)}, \quad B_{h a r}^{O} \geq 0.02 .
$$

These results have been improved slightly by A. Grigoryan [9] and M. Liu [13]. Liu's lower bound for $B_{\text {har }}^{O}$ is 0.0277 .

Now, by a different approach we obtain new lower bounds for $B_{h a r}^{K}$ and $B_{h a r}^{O}$. Denote by $B_{h o l}$ the classical Bloch constant for holomorphic functions.

Theorem 2. $B_{\text {har }}^{K} \geq \frac{2}{K+1} B_{\text {hol }}$, and $B_{\text {har }}^{O} \geq \frac{1}{4} B_{\text {hol }}$. 
Proof. Let $f \in \operatorname{Har}_{K}(D), f_{z}(0)=1$ and $\left|f_{\bar{z}}(0)\right| \leq(K-1) /(K+1)$. Since $f_{z}$ is holomorphic, the integral

$$
\zeta=F(z)=\int_{0}^{z} f_{z}(z) d z
$$

defines a function, holomorphic on $D$, such that $F^{\prime}(0)=1$. Thus, for any $r<B_{\text {hol }}$, there is a disk $\Delta$ of center $\zeta_{0}$ and radius $r$ which is the biholomorphic image of a domain $G \subset D$ under the mapping $F$. The composition $g=f \circ F^{-1}$ is a $K$ quasiregular harmonic mapping. Since $f$ is an interior transformation as indicated in Section $2, f$ is locally univalent outside of a discrete set $E \subset D$ of branch points. As indicated in Section 2, $\operatorname{det} J_{f} \neq 0$ on $D \backslash E$ and, consequently, $\operatorname{det} J_{f}>0$ on $D \backslash E$ since $\operatorname{det} J_{f}(0)>0$. Thus, $\left|f_{z}\right| \geq\left|f_{\bar{z}}\right|$ and

$$
\left|f_{\bar{z}}\right| \leq \frac{K-1}{K+1}\left|f_{z}\right|
$$

holds for $z \in D$.

We have, for $\zeta \in \Delta$,

$$
g_{\zeta}=f_{z} / F^{\prime}(z)=1, \quad g_{\bar{\zeta}}=f_{\bar{z}} / \overline{f_{z}}, \quad\left|g_{\bar{\zeta}}\right| \leq \frac{K-1}{K+1} .
$$

Thus, for $\zeta_{1}, \zeta_{2} \in \Delta$,

$$
\left|g\left(\zeta_{1}\right)-g\left(\zeta_{2}\right)\right| \geq\left|\int_{\overline{\zeta_{1}, \zeta_{2}}} g_{\zeta} d \zeta\right|-\left|\int_{\overline{\zeta_{1}, \zeta_{2}}} g_{\bar{\zeta}} d \bar{\zeta}\right| \geq \frac{2\left|\zeta_{1}-\zeta_{2}\right|}{K+1} .
$$

This shows that $g$ is univalent on $\Delta$ and, consequently, $f$ is univalent on $G$. Further, for $\zeta \in \Delta$,

$$
\left|g(\zeta)-g\left(\zeta_{0}\right)\right| \geq \frac{2\left|\zeta-\zeta_{0}\right|}{K+1} .
$$

The right side of the above estimate tends to $2 r /(K+1)$ as $\zeta \rightarrow \partial \Delta$. This shows that $g(\Delta)=f(G)$ contains a disk of radius $2 r /(K+1)$. Since $r$ can be close to $B_{h o l}$ arbitrarily, this shows that $b_{f} \geq 2 B_{\text {hol }} /(K+1)$. The first estimate is proved.

Now, let $f \in \operatorname{Har}_{O}(D), f_{z}(0)=1$ and $f_{\bar{z}}(0)=0 . f$ is also an interior transformation as proved in Section 2. As above, letting $E \subset D$ be the discrete set of branch points, we have $\operatorname{det} J_{f}>0$ and $\left|f_{z}\right|>\left|f_{\bar{z}}\right|$ for $z \in D \backslash E$. Thus, the holomorphic function $h=\overline{f_{\bar{z}}} / f_{z}$ has its modulus less than 1 on $D \backslash E$. Note that $E$ is removable and $h(0)=0$. Using the Schwarz lemma, we have $|h(z)| \leq|z|$ and, consequently, $\left|f_{\bar{z}}(z)\right| \leq|z|\left|f_{z}(z)\right|$ for $z \in D$. This show that $f$ is a $K$-quasiregular harmonic mapping, with $K=(1+\rho) /(1-\rho)$, of the disk with center origin and radius $\rho$, and $g(z)=\rho^{-1} f(\rho z), 0<\rho<1$, is a $K$-quasiregular harmonic mapping of the unit disk. Note that $g_{z}(0)=1$. Taking $\rho=1 / 2$ and using the first estimate, we obtain that $b_{f} \geq B_{h o l} / 4$. The second estimate is proved and the proof is complete.

The first lower bound estimate in the above theorem is quite satisfactory, since the lower bound tends to $B_{\text {hol }}$ as $K \rightarrow 1$. Using Ahlfors' [1] lower estimate $B_{\text {hol }} \geq$ $\sqrt{3} / 4$, from the above theorem, we have

$$
B_{\text {har }}^{K} \geq \frac{1}{K+1} \frac{\sqrt{3}}{2}, \quad B_{\text {har }}^{O} \geq \frac{\sqrt{3}}{16} \approx 0.108 .
$$




\section{LANDAU'S THEOREM FOR HARMONIC MAPPINGS}

Let $f$ be a holomorphic function on the unit disk $D$. If $f(0)=0, f^{\prime}(0)=\alpha>0$ and $|f(z)|<1$ for $z \in D$, the classical Landau's theorem asserts that $f$ is univalent in the disk $D_{r_{0}}$ of center 0 and radius

$$
r_{0}=\frac{\alpha}{1+\sqrt{1-\alpha^{2}}}>\frac{\alpha}{2},
$$

and $f\left(D_{r_{0}}\right)$ contains a disk $D_{R_{0}}$ of center 0 and radius $R_{0}=r_{0}^{2}$. In [7], it is proved that Landau's theorem holds also for harmonic mappings with the constants $r_{0}$ and $R_{0}$ replaced by $r_{0}=\pi^{2} \alpha /(16 m)$ and $R_{0}=r_{0} \alpha / 2 \approx \alpha^{2} / 22.2$, where $m \approx 6.85$, under the normalization $f_{z}(0)=\alpha$ and $f_{\bar{z}}(0)=0$. Now we improve this result for open harmonic mappings. To do this, we need the Schwarz-Pick lemma for harmonic mappings: for a harmonic mapping $f$ on the unit disk such that $f(0)=0$ and $|f(z)|<1$ for $z \in D$, it is known [11] that

$$
|f(z)| \leq \frac{4}{\pi} \arctan |z|, \quad z \in D
$$

and

$$
\Lambda_{f}(0) \leq \frac{4}{\pi}
$$

Since the composition $f \circ \phi$ of $f$ and a biholomorphic mapping $\phi$ of $D$ is harmonic, as a consequence of (4.2), if the condition $f(0)=0$ is replaced by $f(z)=0$ for some $z \in D$, we have

$$
\Lambda_{f}(z) \leq \frac{4}{\pi} \frac{1}{1-|z|^{2}}
$$

Moreover, (4.3) is always true 8 for any harmonic self-mapping $f$ of $D$ and any point $z \in D$ even without the assumption $f(z)=0$.

Theorem 3. Let $f$ be a harmonic mapping of $D$ such that $f(0)=0, f_{z}(0)=\alpha>0$ and $f_{\bar{z}}(0)=0$, and $|f(z)|<1$ for $z \in D$. In addition, suppose that $\left|f_{\bar{z}}\right| \leq\left|f_{z}\right|$ on $D$. Then, $f$ is univalent on a domain which contains the origin and whose image is a disk of center 0 and radius

$$
R_{0}=k r^{2}\left(1-r^{\prime} / 2\right)
$$

where

$$
k=\frac{2 \log 3}{\pi}, \quad r^{\prime}=\frac{\alpha}{2 k+\sqrt{4 k^{2}-\alpha^{2}}} .
$$

Proof. By $E$, denote the set of zeros of the holomorphic function $f_{z}$. The function $h(z)=\overline{f_{\bar{z}}} / f_{z}$ is holomorphic and has its modulus not exceeding 1 on $D \backslash E$. Since $E$ is removable, using the Schwarz lemma, we have

$$
\left|f_{\bar{z}}(z)\right| \leq|z|\left|f_{z}(z)\right|, \quad z \in D .
$$

Define $\zeta=F(z)=\int_{0}^{z} f_{z}(z) d z$ for $z \in D$. For $\left|z^{\prime}\right|<1 / 2$, by (4.3), we have

$$
\begin{gathered}
\left|F\left(z^{\prime}\right)\right| \leq \int_{\overline{0, z^{\prime}}}\left|f_{z}(z)\right||d z| \leq \int_{\overline{0, z^{\prime}}} \Lambda_{f}(z)|d z| \\
\leq \frac{4}{\pi} \int_{0}^{\left|z^{\prime}\right|} \frac{d r}{1-r^{2}}=\frac{2}{\pi} \log \frac{1+\left|z^{\prime}\right|}{1-\left|z^{\prime}\right|}<k=\frac{2 \log 3}{\pi} .
\end{gathered}
$$


Using Landau's theorem for $g(z)=k^{-1} F(z / 2)$, we see that $g$ is univalent on the disk $D_{r^{\prime}}$ of center the origin and radius $r^{\prime}$ and $g\left(D_{r^{\prime}}\right)$ contains the disk $D_{R^{\prime}}$ with $R^{\prime}=r^{\prime 2}$. Consequently, $F$ is univalent on $D_{r^{\prime} / 2}$, and $F\left(D_{r^{\prime} / 2}\right)$ contains the disk $D_{k R^{\prime}}$.

Let $G=F_{D_{r^{\prime} / 2}}^{-1}\left(D_{k R^{\prime}}\right) \subset D_{r^{\prime} / 2}$. For $z_{1}, z_{2} \in G$, let $\gamma=F^{-1}\left(\overline{F\left(z_{1}\right), F\left(z_{2}\right)}\right)$. Then,

$$
\left|F\left(z_{2}\right)-F\left(z_{1}\right)\right|=\left|\int_{\gamma} f_{z}(z) d z\right|=\int_{\gamma}\left|f_{z}(z)\right||d z| .
$$

We have, by (4.4),

$$
\begin{gathered}
\left|f\left(z_{1}\right)-f\left(z_{2}\right)\right| \geq\left|\int_{\gamma} f_{z}(z) d z\right|-\left|\int_{\gamma} f_{\bar{z}}(z) d \bar{z}\right| \\
\geq\left|F\left(z_{2}\right)-F\left(z_{1}\right)\right|-\int_{\gamma}|z|\left|f_{z}(z)\right||d z| \geq\left(1-\frac{r^{\prime}}{2}\right)\left|F\left(z_{2}\right)-F\left(z_{1}\right)\right|,
\end{gathered}
$$

from which we see that $f$ is univalent on $G$ since $r^{\prime}<1$. Further, $f(0)=0$ and $|f(z)| \geq\left(1-r^{\prime} / 2\right)|F(z)| \rightarrow k R^{\prime}\left(1-r^{\prime} / 2\right)$ as $z \rightarrow \partial G$. This shows that $f(G)$ contains the disk of center the origin and radius $k R^{\prime}\left(1-r^{\prime} / 2\right)$. The theorem is proved.

We notice that $R_{0} \approx \alpha^{2} /(16 k) \approx \alpha^{2} / 11.2$ as $\alpha \rightarrow 0$. As in the proof of Theorem 2, under the normalization in Theorem 3, the openness of $f$ implies that $\left|f_{\bar{z}}\right| \leq\left|f_{z}\right|$ on $D$. So, Theorem 3 holds if the additional assumption is replaced by the openness of $f$.

\section{ThE SCHWARZ-PICK LEMMA FOR PLURIHARMONIC MAPPINGS}

In this section, we prove that (4.3), without the assumption $f(z)=0$, holds also for any pluriharmonic mapping $f$ of the unit ball $B^{n}$ into the complex space $\mathbb{C}^{n}$ of dimension $n$. A continuous complex-valued function $\phi$ defined on a domain $\Omega \subset \mathbb{C}^{n}$ is said to be pluriharmonic if, for each fixed $z^{\prime} \in \Omega$ and $\theta \in \partial B^{n}$, the function $\phi\left(z^{\prime}+\theta \zeta\right)$ is harmonic in the complex variable $\zeta$, for $|\zeta|$ smaller than the distance of $z^{\prime}$ from $\partial \Omega$. A mapping $f$ of $\Omega$ into $\mathbb{C}^{n}$ is said to be pluriharmonic if every component of $f$ is pluriharmonic. A mapping $f$ of $B^{n}$ into $\mathbb{C}^{n}$ is pluriharmonic if and only if $f$ has a representation $f=g+\bar{h}$, where $g$ and $h$ are holomorphic mappings.

For a continuously differentiable mapping $w=f(z)=\left(f_{1}(z), \cdots, f_{m}(z)\right), z=$ $\left(z_{1}, \cdots, z_{n}\right)$, of a domain $\Omega \subset \mathbb{C}^{n}$ into $\mathbb{C}^{m}$, by $f_{z}$ and $f_{\bar{z}}$ denote the matrices $\left(\partial f_{j} / \partial z_{k}\right)_{m \times n}$ and $\left(\partial f_{j} / \partial \bar{z}_{k}\right)_{m \times n}$ respectively. Denote the maximum dilation and minimum dilation by

$$
\Lambda_{f}=\max _{\theta \in \partial \mathbb{B}^{n}}\left|f_{z} \theta+f_{\bar{z}} \bar{\theta}\right|, \quad \lambda_{f}=\min _{\theta \in \partial \mathbb{B}^{n}}\left|f_{z} \theta+f_{\bar{z}} \bar{\theta}\right| .
$$

Here, $\theta$ is regarded as a column vector. From now on, we identify a point in $\mathbb{C}^{n}$ or $\mathbb{R}^{n}$ (real space of dimension $n$ ) with a complex or real column vector.

Theorem 4. Let $f$ be a pluriharmonic mapping of $B^{n}$ into $B^{m}$. Then

$$
\Lambda_{f}(z) \leq \frac{4}{\pi} \frac{1}{1-|z|^{2}} \quad \text { for } \quad z \in B^{n}
$$

If $f(0)=0$, then

$$
|f(z)| \leq \frac{4}{\pi} \arctan |z| \quad \text { for } \quad z \in B^{n}
$$


Proof. To prove (5.1), consider $z=0$ first. Let $\Lambda_{f}(0)=\left|f_{z}(0) \theta^{\prime}+f_{\bar{z}}(0) \overline{\theta^{\prime}}\right|$ and $\omega=f_{z}(0) \theta^{\prime}+f_{\bar{z}}(0) \overline{\theta^{\prime}} \neq 0$. Define the harmonic function $\phi(\zeta)=\left\langle f\left(\zeta \theta^{\prime}\right), \omega /|\omega|\right\rangle$ for $\zeta \in D$. Then, $|\phi(\zeta)| \leq 1$ for $\zeta \in D$ and $\phi_{\zeta}(0)+\phi_{\bar{\zeta}}(0)=\langle\omega, \omega /|\omega|\rangle=|\omega|$. Since $\phi$ is harmonic, (4.3) holds for $\phi$. Thus,

$$
\Lambda_{f}(0)=|\omega|=\left|\phi_{\zeta}(0)+\phi_{\bar{\zeta}}(0)\right| \leq \Lambda_{\phi}(0) \leq \frac{4}{\pi} .
$$

This shows (5.1) for $z=0$.

Let $z^{\prime} \in B^{n}$ be fixed. By $\varphi_{z^{\prime}}$ we denote the Möbius transformation of $B^{n}$ such that $\varphi\left(z^{\prime}\right)=0$ and $\varphi_{z^{\prime}}=\varphi_{z^{\prime}}^{-1}$; see [15. It is known that

$$
\Lambda_{\varphi_{z^{\prime}}}\left(z^{\prime}\right)=\frac{1}{1-\left|z^{\prime}\right|^{2}}
$$

By what was proved above, we have $\Lambda_{f \circ \varphi_{z^{\prime}}}(0) \leq 4 / \pi$ and, consequently,

$$
\Lambda_{f}\left(z^{\prime}\right) \leq \Lambda_{\varphi_{z^{\prime}}}\left(z^{\prime}\right) \Lambda_{f \circ \varphi_{z^{\prime}}}(0) \leq \frac{4}{\pi} \frac{1}{1-\left|z^{\prime}\right|^{2}} .
$$

This shows (5.1) completely.

For any fixed $z^{\prime} \in B^{n} \backslash\{0\}$, letting $\phi(\zeta)=\left\langle f\left(\zeta z^{\prime} /\left|z^{\prime}\right|\right), f\left(z^{\prime}\right) /\left|f\left(z^{\prime}\right)\right|\right\rangle$ for $\zeta \in D$ and using (4.1), we obtain $\left|f\left(z^{\prime}\right)\right|=\left|\phi\left(\left|z^{\prime}\right|\right)\right| \leq(4 / \pi) \arctan \left|z^{\prime}\right|$. This shows (5.2), and the lemma is proved.

\section{The LANDAU THEOREM FOR PLURIHARMONIC MAPPINGS}

The following Landau theorem for holomorphic mappings of $B^{n}$ into $\mathbb{C}^{n}$ was proved in [6]: Let $f$ be a holomorphic mapping of $B^{n}$ into $\mathbb{C}^{n}$ such that $f(0)=0$, $\operatorname{det} f^{\prime}(0)=\alpha>0$, and $|f(z)|<M$ for $z \in B^{n}$. Let

$$
\rho_{0}=\frac{\alpha}{m M^{n}}, \quad \rho_{1}=\frac{\alpha}{2 M^{n}}, \quad R_{0}=M \rho_{0} \rho_{1}=\frac{\alpha^{2}}{2 m M^{2 n-1}},
$$

where $m \approx 4.2$ is the minimum of the function $\left(2-r^{2}\right) /\left(r\left(1-r^{2}\right)\right)$ for $0 \leq r \leq 1$. Then $f$ maps $B^{n}\left(0, \rho_{0}\right)$ injectively onto a domain which contains $B^{n}\left(0, R_{0}\right)$. Here, by $B^{n}(z, r)$ we denote the ball in $\mathbb{C}^{n}$ of center $z$ and radius $r$. In this section, we establish the Landau theorem for pluriharmonic mappings.

For a continuously differentiable mapping $w=f(z)=\left(f_{1}(z), \cdots, f_{n}(z)\right), z=$ $\left(z_{1}, \cdots, z_{n}\right)$, of a domain $\Omega \subset \mathbb{C}^{n}$ into $\mathbb{C}^{n}$, let $z_{k}=x_{k}+i y_{k}$ and $f_{j}=u_{j}+i v_{j} . f$ can be regarded as a mapping of a domain in $\mathbb{R}^{2 n}$ into $\mathbb{R}^{2 n}$. We denote the real Jacobian matrix of this mapping by

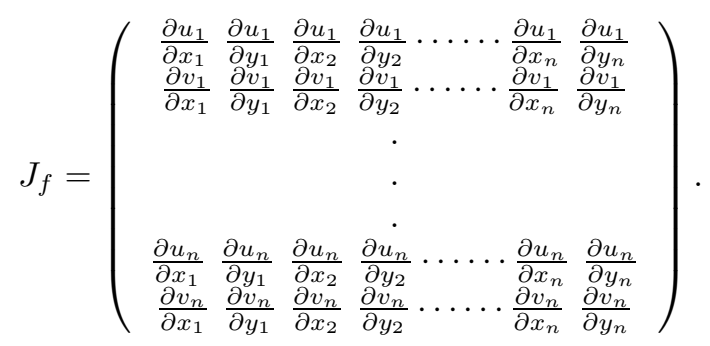

Let $\mathbb{B}^{2 n}$ denote the unit ball in $\mathbb{R}^{2 n}$. With this notation, the maximum dilation and minimum dilation $\Lambda_{f}$ and $\lambda_{f}$ have another expression:

$$
\Lambda_{f}=\max _{\theta \in \partial \mathbb{B}^{2 n}}\left|J_{f} \theta\right|, \quad \lambda_{f}=\min _{\theta \in \partial \mathbb{B}^{2 n}}\left|J_{f} \theta\right| .
$$


For a complex or real $n \times n$ matrix $A$, the operator norm of $A$ is defined by

$$
|A|=\max _{\theta \in \partial B^{n}}|A \theta| \quad \text { or }|A|=\max _{\theta \in \partial \mathbb{B}^{n}}|A \theta| .
$$

Note that $\Lambda_{f}=\left|J_{f}\right|$.

Lemma 1. Let $A$ be an $n \times n$ complex (real) matrix. Then, for any unit vector $\theta \in \partial B^{n}\left(\partial \mathbb{B}^{n}\right)$, the following inequality holds:

$$
|A| \geq|\operatorname{det}(A)|^{1 / n}, \quad|A \theta| \geq \frac{|\operatorname{det}(A)|}{|A|^{n-1}} .
$$

If $A$ satisfies $|A| \leq K|\operatorname{det}(A)|^{1 / n}, K \geq 1$, then, for any $\theta \in \partial B^{n}\left(\partial \mathbb{B}^{n}\right)$, we have

$$
|A \theta| \geq \frac{|\operatorname{det}(A)|^{1 / n}}{K^{n-1}} .
$$

Proof. First assume that $A$ is a complex matrix. We may assume that $\operatorname{det}(A) \neq 0$. There exist two unitary matrices $U_{1}$ and $U_{2}$ such that $\Lambda=U_{1} A U_{2}$ is a diagonal matrix with positive elements $\lambda_{1}, \lambda_{2}, \cdots, \lambda_{n}$. Then

$$
|\operatorname{det}(A)|=\lambda_{1} \lambda_{2} \cdots \lambda_{n} \text {. }
$$

Assume that $0<\lambda_{1} \leq \lambda_{2} \leq \cdots \leq \lambda_{n}$. For any $\theta \in \partial B^{n}$, since $U_{1}$ and $U_{2}$ are unitary, we have

$$
|A \theta|=\left|U_{1} A U_{2} U_{2}^{-1} \theta\right|=|\Lambda \omega| \geq \lambda_{1}=\frac{|\operatorname{det}(A)|}{\lambda_{2} \cdots \lambda_{n}},
$$

where $\omega=U_{2}^{-1} \theta \in \partial B^{n}$. Let $\omega$ be the unit vector, of which only the $k$-th component is not zero. Then, since $U_{1}$ is unitary,

$$
\lambda_{k}=|\Lambda \omega|=\left|U_{1} A U_{2} \omega\right|=\left|A U_{2} \omega\right| .
$$

Also, since $U_{2}$ is unitary, $\omega^{\prime}=U_{2} \omega$ is also a unit vector since $U_{2}$ is unitary. Thus,

$$
\lambda_{k}=\left|A \omega^{\prime}\right| \leq|A|,
$$

and, if $|A| \leq K|\operatorname{det}(A)|^{1 / n}$, we have

$$
\lambda_{k} \leq K|\operatorname{det}(A)|^{1 / n} .
$$

This shows the lemma for a complex matrix. The proof is completely the same in the real case.

As a consequence of (6.1), for a continuously differentiable mapping $f$ of a domain $\Omega \subset \mathbb{C}^{n}$ into $\mathbb{C}^{n}$, we have

$$
\lambda_{f} \geq\left|\operatorname{det} J_{f}\right| / \Lambda_{f}^{2 n-1} .
$$

Theorem 5. Let $f$ be a pluriharmonic mapping of $B^{n}$ into $\mathbb{C}^{n}$ such that $f(0)=0$, $|f(z)|<M$ for $z \in B^{n}$ and $(4 M / \pi)^{2 n} \geq \operatorname{det} J_{f}(0)=\alpha>0$. Let

$$
\rho_{0}=\frac{\alpha \pi^{2 n+1}}{4 m(4 M)^{2 n}}, \quad R_{0}=\frac{\alpha^{2} \pi^{4 n}}{8 m(4 M)^{4 n-1}},
$$

where $m \approx 4.2$ is the minimum of the function $\frac{2-r^{2}}{r\left(1-r^{2}\right)}$ on the interval $(0,1)$. Then $f$ is univalent on the ball $B^{n}\left(0, \rho_{0}\right)$, and $f\left(B^{n}\left(0, \rho_{0}\right)\right)$ covers the ball $B^{n}\left(0, R_{0}\right)$. 
Proof. The function $\frac{2-r^{2}}{r\left(1-r^{2}\right)}$ attains its minimum $m \approx 4.2$ at $r_{0} \approx 0.66$. For fixed different points $z^{\prime}, z^{\prime \prime} \in B^{n}\left(0, \rho_{0}\right)$, let $z^{\prime \prime}-z^{\prime}=\left|z^{\prime \prime}-z^{\prime}\right| \theta$ and define the pluriharmonic mapping

$$
\phi_{\theta}(z)=\left(f_{z}(z)-f_{z}(0)\right) \theta+\left(f_{\bar{z}}(z)-f_{\bar{z}}(0)\right) \bar{\theta} .
$$

Then, by (5.1),

$$
\left|\phi_{\theta}(z)\right| \leq \Lambda_{f}(0)+\Lambda_{f}(z) \leq \frac{4 M}{\pi}\left(1+\frac{1}{1-|z|^{2}}\right)<\frac{4 M}{\pi} \frac{2-r_{0}^{2}}{1-r_{0}^{2}} \quad \text { for }|z|<r_{0} .
$$

Consequently, using (5.2), we obtain

$$
\left|\phi_{\theta}(z)\right| \leq \frac{16 M|z|}{\pi^{2}} \frac{2-r_{0}^{2}}{r_{0}\left(1-r_{0}^{2}\right)}=\frac{16 m M|z|}{\pi^{2}} \text { for }|z|<r_{0} .
$$

We have

$$
\begin{gathered}
\left|f\left(z^{\prime \prime}\right)-f\left(z^{\prime}\right)\right|=\left|\int_{\overline{z^{\prime} z^{\prime \prime}}} f_{z}(z) d z+f_{\bar{z}}(z) d \bar{z}\right| \\
\geq\left|\int_{\overline{z^{\prime} z^{\prime \prime}}} f_{z}(0) d z+f_{\bar{z}}(0) d \bar{z}\right|-\left|\int_{\overline{z^{\prime} z^{\prime \prime}}}\left(f_{z}(z)-f_{z}(0)\right) d z+\left(f_{\bar{z}}(z)-f_{\bar{z}}(0)\right) d \bar{z}\right|, \\
\left|\int_{\overline{z^{\prime} z^{\prime \prime}}} f_{z}(0) d z+f_{\bar{z}}(0) d \bar{z}\right| \geq\left|f_{z}(0) \theta+f_{\bar{z}}(0) \bar{\theta}\right| \int_{\overline{z^{\prime} z^{\prime \prime}}} d s \\
\geq \lambda_{f}(0)\left|z^{\prime \prime}-z^{\prime}\right| \geq \frac{\left|\operatorname{det} J_{f}(0)\right|}{\Lambda_{f}(0)^{2 n-1}}\left|z^{\prime \prime}-z^{\prime}\right| \geq \frac{\alpha \pi^{2 n-1}}{(4 M)^{2 n-1}}\left|z^{\prime \prime}-z^{\prime}\right|,
\end{gathered}
$$

where (6.3) and (5.1) are used, and

$$
\begin{aligned}
& \left|\int_{\overline{\bar{z}^{\prime} \bar{z}^{\prime \prime}}}\left(f_{z}(z)-f_{z}(0)\right) d z+\left(f_{\bar{z}}(z)-f_{\bar{z}}(0)\right) d \bar{z}\right| \\
& \leq \int_{\overline{z^{\prime} z^{\prime \prime}}}\left|\left(f_{z}(z)-f_{z}(0)\right) \theta+\left(f_{\bar{z}}(z)-f_{\bar{z}}(0)\right) \bar{\theta}\right| d s \\
& \quad<\frac{16 m M \rho_{0}}{\pi^{2}}\left|z^{\prime \prime}-z^{\prime}\right|=\frac{\alpha \pi^{2 n-1}}{(4 M)^{2 n-1}}\left|z^{\prime \prime}-z^{\prime}\right|,
\end{aligned}
$$

since $\rho_{0} \leq \pi /(4 m)<r_{0}$. Thus $f\left(z^{\prime \prime}\right) \neq f\left(z^{\prime}\right)$. This shows that $f$ is univalent in $B^{n}\left(0, \rho_{0}\right)$.

Now, let $z^{\prime} \in \partial B^{n}\left(0, \rho_{0}\right)$. We have

$$
\begin{aligned}
\left|f\left(z^{\prime}\right)\right| & =\left|\int_{\overline{0 z^{\prime}}} f_{z}(z) d z+f_{\bar{z}}(z) d \bar{z}\right| \\
& \geq\left|\int_{\overline{0 z^{\prime}}} f_{z}(0) d z+f_{\bar{z}}(0) d \bar{z}\right|-\left|\int_{\overline{0 z^{\prime}}}\left(f_{z}(z)-f_{z}(0)\right) d z+\left(f_{\bar{z}}(z)-f_{\bar{z}}(0)\right) d \bar{z}\right| \\
& \geq \frac{\alpha^{2} \pi^{4 n}}{4 m(4 M)^{4 n-1}}-\int_{0}^{\rho_{0}} \frac{16 m M r}{\pi^{2}} d r=\frac{\alpha^{2} \pi^{4 n}}{8 m(4 M)^{4 n-1}} .
\end{aligned}
$$

This shows that $f\left(B^{n}\left(0, \rho_{0}\right)\right)$ covers the ball $B^{n}\left(0, R_{0}\right)$. The theorem is proved.

\section{The Bloch THEOREM FOR PLURIHARMONIC MAPPINGS}

In the case of several variables, the notions of univalent ball and inradius $b_{f}$ are defined in a similar way as that in one variable. For general holomorphic mappings of more than one complex variable, there is no (positive) Bloch constant ([10], [18); in order to obtain higher dimensional analogs of Bloch's theorem, it is necessary to 
restrict the class of mappings considered. In [18, H. Wu defined the quasiregularity of a holomorphic mapping in a way which is a little different from that in Section 3 of this paper. A holomorphic mapping $f$ of $B^{n}$ into $\mathbb{C}^{n}$ is said to be a Wu $K$-mapping, $K \geq 1$, if

$$
\left|f^{\prime}(z)\right| \leq K\left|\operatorname{det} f^{\prime}(z)\right|^{1 / n} \quad \text { for } \quad z \in B^{n},
$$

where $f^{\prime}(z)$ is the Jacobian matrix of the mapping $f$ at $z$, and $\left|f^{\prime}(z)\right|$ is the operator norm which is just the maximum dilation $\Lambda_{f}(z)$. For a Wu $K$-mapping of the unit ball $B^{n}$ into $\mathbf{C}^{n}, n>1$, such that $\operatorname{det} f^{\prime}(0)=1$, the authors of the present paper [6] gave two lower bounds of the inradius $b_{f}$ :

$$
b_{f} \geq \frac{1}{10 K^{2 n-1}}, \quad b_{f} \geq \frac{1}{12 K^{n-1}} .
$$

Now, we generalize the notion of Wu $K$-mapping to pluriharmonic mappings and establish a lower bound estimate of the Bloch constant for such mappings.

A pluriharmonic mapping $f$ of $B^{n}$ into $\mathbb{C}^{n}$ is said to be a $K$-mapping if

$$
\left|J_{f}(z)\right| \leq K\left|\operatorname{det} J_{f}(z)\right|^{1 /(2 n)} \quad \text { for } z \in B^{n},
$$

where $J_{f}$ is defined in Section 8. For pluriharmonic $K$-mappings, we have the following Bloch theorem.

Theorem 6. Let $f$ be a pluriharmonic $K$-mapping of the unit ball $B^{n}$ into $\mathbb{C}^{n}$, $n>1$, such that $\operatorname{det} J_{f}(0)=1$. Then

$$
b_{f} \geq R_{n}=\frac{k_{n} \pi}{8 m}\left(\frac{k_{n} \pi}{4 K \log \left(1 /\left(1-k_{n}\right)\right)}\right)^{4 n-1},
$$

where $0<k_{n}<1$ is the unique number such that

$$
4 n \log \frac{1}{1-k_{n}}=(4 n-1) \cdot \frac{k_{n}}{1-k_{n}} .
$$

Proof. Without loss of generality, we may assume that $f$ is pluriharmonic on $\bar{B}^{n}$. Let $z^{\prime} \in B^{n}$ be a point such that

$$
\left(1-\left|z^{\prime}\right|\right)^{2 n}\left|\operatorname{det} J_{f}\left(z^{\prime}\right)\right|=1
$$

and

$$
(1-|z|)^{2 n}\left|\operatorname{det} J_{f}(z)\right| \leq 1 \quad \text { for }\left|z^{\prime}\right|=r \leq|z| \leq 1 .
$$

In particular,

$$
\left|\operatorname{det} J_{f}(z)\right| \leq\left|\operatorname{det} J_{f}\left(z^{\prime}\right)\right| \text { for }|z|=r .
$$

Let $\left|z_{0}\right| \leq r$. Assume that $\Lambda_{f}\left(z_{0}\right)=\left|f_{z}\left(z_{0}\right) \theta+f_{\bar{z}}\left(z_{0}\right) \bar{\theta}\right|$ with $\theta \in \partial B^{n}$. Define $\phi(\zeta)=f_{z}\left(\zeta z_{0} /\left|z_{0}\right|\right) \theta+f_{\bar{z}}\left(\zeta z_{0} /\left|z_{0}\right|\right) \bar{\theta}$ for $\zeta \in D$. Since $\phi$ is harmonic, by the maximum principle,

$$
\Lambda_{f}\left(z_{0}\right)=\left|\phi\left(\left|z_{0}\right|\right)\right| \leq\left|f_{z}\left(\zeta^{\prime} z_{0} /\left|z_{0}\right|\right) \theta+f_{\bar{z}}\left(\zeta^{\prime} z_{0} /\left|z_{0}\right|\right) \bar{\theta}\right|
$$

with $\left|\zeta^{\prime}\right|=r$. Let $z^{\prime \prime}=\zeta^{\prime} z_{0} /\left|z_{0}\right|$. Note that $\left|z^{\prime \prime}\right|=r$. Thus, by (7.4), and the definition of $K$-mappings,

$$
\begin{aligned}
\Lambda_{f}\left(z_{0}\right) & \leq\left|f_{z}\left(z^{\prime \prime}\right) \theta+f_{\bar{z}}\left(z^{\prime \prime}\right) \bar{\theta}\right| \\
& \leq \Lambda_{f}\left(z^{\prime \prime}\right)=\left|J_{f}\left(z^{\prime \prime}\right)\right| \leq K\left|\operatorname{det} J_{f}\left(z^{\prime \prime}\right)\right|^{1 /(2 n)} \leq K\left|\operatorname{det} J_{f}\left(z^{\prime}\right)\right|^{1 /(2 n)} .
\end{aligned}
$$

This shows that

$$
\Lambda_{f}(z) \leq K\left|\operatorname{det} J_{f}\left(z^{\prime}\right)\right|^{1 /(2 n)} \text { for }|z| \leq r .
$$


For $\zeta \in B^{n}$, define

$$
g(\zeta)=z^{\prime}+k(1-r) \zeta, \quad F(\zeta)=\frac{1}{k}\left(f(g(\zeta))-f\left(z^{\prime}\right)\right),
$$

where $k=k_{n}$. Then,

$$
F(0)=0, \quad\left|\operatorname{det} J_{F}(0)\right|=(1-r)^{2 n}\left|\operatorname{det} J_{f}\left(z^{\prime}\right)\right|=1 .
$$

If $|g(\zeta)| \leq r$, by $(7.5)$ and $(7.2)$,

$$
\begin{gathered}
\Lambda_{F}(\zeta)=(1-r) \Lambda_{f}(g(\zeta)) \\
\leq K(1-r)\left|\operatorname{det} J_{f}\left(z^{\prime}\right)\right|^{1 /(2 n)}=K,
\end{gathered}
$$

and if $|g(\zeta)| \geq r$,

$$
\begin{aligned}
\Lambda_{F}(\zeta) & =(1-r) \Lambda_{f}(g(\zeta)) \leq K(1-r)\left|\operatorname{det} J_{f}(g(\zeta))\right|^{1 /(2 n)} \\
& =K\left(\frac{1-r}{1-|g(\zeta)|}\right)(1-|g(\zeta)|)\left|\operatorname{det} J_{f}(g(\zeta))\right|^{1 /(2 n)} \leq K\left(\frac{1-r}{1-|g(\zeta)|}\right) \\
& \leq K \cdot \frac{1-r}{1-r-k(1-r)|\zeta|}=\frac{K}{1-k|\zeta|} .
\end{aligned}
$$

We conclude that

$$
\Lambda_{F}(\zeta) \leq \frac{K}{1-k|\zeta|} \quad \text { for } \zeta \in B^{n} .
$$

Thus, for $\zeta^{\prime} \in B^{n}$, we have

$$
\left|F\left(\zeta^{\prime}\right)\right| \leq \int_{\overline{0 \zeta^{\prime}}}|d F(\zeta)|=\int_{\overline{0 \zeta^{\prime}}} \Lambda_{F}(\zeta) d s \leq K \int_{0}^{\left|\zeta^{\prime}\right|} \frac{d s}{1-k s}<\frac{K}{k} \log \frac{1}{1-k} .
$$

Now, applying Theorem 5 to the function $F$, we see that $F\left(B^{n}\right)$ contains a schlicht ball with center 0 and radius

$$
\frac{\pi}{8 m}\left(\frac{k \pi}{4 K \log (1 /(1-k))}\right)^{4 n-1} .
$$

Consequently, $f\left(B^{n}\right)$ contains the schlicht ball with center $f\left(z^{\prime}\right)$ and radius $R_{n}$. This proves the theorem.

Now, we give an approximative estimate for $R_{n}$. Because of (7.1), we have

$$
1=(2 n-1) k_{n}+(4 n-1-4 n / 3) k_{n}^{2}+\cdots,
$$

from which it follows that $k_{n}<\frac{1}{2 n-1}$ and

$$
k_{n} \geq \frac{1}{2 n-1}\left(1-\frac{4 n-1}{2 n-1} \frac{k_{n}}{1-k_{n}}\right)=\frac{1}{2 n-1}(1-o(1)) .
$$

Replacing $\log \frac{1}{1-k_{n}}$ by $\frac{4 n-1}{4 n} \frac{k_{n}}{1-k_{n}}$ in the formula of $R_{n}$ gives

$$
R_{n}=\frac{\pi}{8 m}\left(\frac{\pi}{4}\right)^{4 n-1} \frac{1}{K^{4 n-1}}\left(\frac{4 n}{4 n-1}\right)^{4 n-1} k_{n}\left(1-k_{n}\right)^{4 n-1} .
$$

Since

$$
\left(\frac{4 n}{4 n-1}\right)^{4 n-1}=\left(1+\frac{1}{4 n-1}\right)^{4 n-1} \rightarrow e, \quad k_{n}=\frac{1}{2 n-1}(1+o(1)),
$$

and

$$
\left(1-k_{n}\right)^{4 n-1}=\left[\left(1-k_{n}\right)^{1 / k_{n}}\right]^{(4 n-1) k_{n}} \rightarrow \frac{1}{e^{2}},
$$


we have

$$
R_{n}=\frac{\pi}{8 m e} \frac{1}{2 n-1}\left(\frac{\pi}{4}\right)^{4 n-1} \frac{1}{K^{4 n-1}}(1+o(1)) .
$$

For locally univalent pluriharmonic $K$-mappings, Theorem 5 has another version and Theorem 6 can be improved. It is known 14 that for $n>1$, a quasiregular holomorphic mapping is locally biholomorphic. However, it is not true that a pluriharmonic $K$-mapping is always locally univalent.

Theorem 7. Let $f$ be a locally univalent pluriharmonic K-mapping of the unit ball $B^{n}$ into $\mathbb{C}^{n}, n>1$, such that $f(0)=0,(4 M / \pi)^{2 n} \geq \operatorname{det} J_{f}(0)=\alpha>0$, and $|f(z)|<M$ for $z \in B^{n}$. Then there exists a domain $\Omega \subset B^{n}\left(0, \rho_{0}\right)$ such that $0 \in \Omega$ and $f$ maps $\Omega$ onto a ball $B^{n}\left(0, R_{0}\right)$ injectively, where

$$
\rho_{0}=\frac{\pi^{2} \alpha^{1 /(2 n)}}{16 m M K^{2 n-1}}, \quad R_{0}=\frac{\pi^{2} \alpha^{1 / n}}{32 m M K^{4 n-2}},
$$

and $m$ is the same number as in Theorem 5.

Proof. For any fixed $z^{\prime}$ with $\left|z^{\prime}\right|=\rho_{0}$, letting $\theta=z^{\prime} /\left|z^{\prime}\right|$ and using (6.2) and (6.4), we have

$$
\begin{aligned}
\left|f\left(z^{\prime}\right)\right| & =\left|\int_{\overline{0 z^{\prime}}} f_{z}(z) d z+f_{\bar{z}}(z) d \bar{z}\right| \\
& \geq\left|\int_{\overline{0 z^{\prime}}} f_{z}(0) d z+f_{\bar{z}}(0) d \bar{z}\right|-\left|\int_{\overline{0 z^{\prime}}}\left(f_{z}(z)-f_{z}(0)\right) d z+\left(f_{\bar{z}}(z)-f_{\bar{z}}(0)\right) d \bar{z}\right| \\
& \geq \lambda_{f}(0) \rho_{0}-\left|\int_{0}^{\rho_{0}} \phi_{\theta}(z) d r\right| \geq \frac{\alpha^{1 / 2 n} \rho_{0}}{K^{2 n-1}}-\int_{0}^{\rho_{0}} \frac{16 m M r}{\pi^{2}} d r \\
& =\frac{\alpha^{1 /(2 n)} \rho_{0}}{K^{2 n-1}}-\frac{8 m M r \rho_{0}^{2}}{\pi^{2}}=R_{0} .
\end{aligned}
$$

Denote $B=B^{n}\left(0, R_{0}\right), B^{\prime}=B^{n}\left(0, \rho_{0}\right)$, and let $\Omega$ be a component of $f_{B^{\prime}}^{-1}(B)$ which contains 0 . It follows from what we proved above that $f$ makes $\Omega$ a regular covering space of $B$. We recall that a covering is said to be regular if the mapping is locally topological and the lifting is always possible. Thus, by the monodromy theorem in the theory of covering spaces, $f$ maps $\Omega$ onto $B$ topologically. The theorem is proved.

If replacing Theorem 5 we use Theorem 7 at the last step of the proof of Theorem 6 and let $k=0.7$, then we obtain the following Bloch theorem.

Theorem 8. Let $f$ be a locally univalent pluriharmonic $K$-mapping of the unit ball $B^{n}$ into $\mathbb{C}^{n}, n>1$, such that $\operatorname{det} J_{f}(0)=1$. Then

$$
b_{f} \geq \frac{1}{134 K^{4 n-1}} .
$$

\section{REFERENCES}

[1] L. V. Ahlfors, An extension of Schwarz's lemma, Trans. Amer. Math. Soc. 43 (1938), 359-364. MR.1501949

[2] S. Bochner, Bloch's theorem for real variables, Bull. Amer. Math. Soc. 52 (1946), 715-719. MR0017814 (8:204a)

[3] M. Bonk, On Bloch's constant, Proc. Amer. Math. Soc. 110 (1990), 889-894. MR979048 (91c:30011)

[4] D. Bshouty and W. Hengartner, Univalent harmonic mappings in the plane, Ann. Univ. Mariae Curie-Sklodowska Sect. A 48 (1994), 12-42. MR.1346560(96m:30025) 
[5] H. Chen and P. M. Gauthier, On Bloch's constant, J. Anal. Math. 69 (1996), 275-291. MR:1428103 (97j:30002)

[6] H. Chen and P. M. Gauthier, Bloch constants in several variables, Trans. Amer. Math. Soc. 353 (2001), 1371-1386. MR1806737 (2001m:32035)

[7] H. Chen, P. M. Gauthier and W. Hengartner, Bloch constants for planar harmonic mappings. Proc. Amer. Math. Soc. 128 (2000), 3231-3240. MR.1707142 (2001b:30027)

[8] F. Colonna, The Bloch constant of bounded harmonic mappings, Indiana Univ. Math. J. 38 (1989), 829-840. MR1029679 (90k:31002)

[9] A. Grigoryan, Landau and Bloch theorems for harmonic mappings, Complex Variable Theory Appl., 51 (2006), 81-87. MR2201260(2006i:30026)

[10] L. A. Harris, On the size of balls covered by analytic transformations, Monatsh. Math. 83 (1977), 9-23. MR0435454 (55:8414)

[11] E. Heinz, On one-to-one harmonic mappings, Pacific J. Math. 9 (1959), 101-105. MR.0104933 $(21: 3683)$

[12] H. Lewy, On the non-vanishing of the Jacobian in certain one-to-one mappings, Bull. Amer. Math. Soc. 42 (1936), 689-692. MR1563404

[13] M. Liu, Estimates on Bloch constants for planar harmonic mappings, Science in China Series A: Mathematics 52 (2009), 87-93. MR2471518 (2010a:30036)

[14] A. Marden and S. Rickman, Holomorphic mappings of bounded distortion, Proc. Amer. Math. Soc. 46 (1974), 225-228. MR0348146 (50:644)

[15] W. Rudin, Function Theory in the Unit Ball of $\mathbf{C}^{n}$, Springer-Verlag, New York, Heidelberg, Berlin, 1980, 23-30. MR601594 (82i:32002)

[16] S. Stoïlow, Leçons sur les principes topologiques de la théorie des fonctions analytiques, Gauthier-Villars, Paris, 1938. MR0082545 (18:568b)

[17] J. C. Wood, Lewy's theorem fails in higher dimensions, Math. Scand. 69 (1991), 166. MR.1156423 (93a:58024)

[18] H. Wu, Normal families of holomorphic mappings, Acta Math. 119 (1967), 193-233. MR0224869 (37:468)

Department of Mathematics, Nanjing Normal University, Nanjing, Jiangsu, 210097, People's Republic of ChinA

E-mail address: hhchen@njnu.edu.cn

Département de Mathématiques et de Statistique, Université de Montréal, CP 6128Centreville, Montreal, QC, H3C 3J7 Canada

E-mail address: gauthier@dms.umontreal.ca 